\title{
Erratum: Cellular Uptake of Elastic Nanoparticles [Phys. Rev. Lett. 107, 098101 (2011)]
}

\author{
Xin Yi, Xinghua Shi, and Huajian Gao
}

(Received 15 October 2018; published 7 November 2018)

DOI: 10.1103/PhysRevLett.121.199902

In determining the wrapping phase diagrams in Fig. 3, we needed to calculate the slope of the energy profile $\Delta E$ with respect to the contact area, or equivalently to the wrapping degree $f$. In our numerical scheme, we interpolated the numerical results of $\Delta E(f)$ by a cubic spline algorithm and calculated the slope $d(\Delta E) / d f$ at different normalized membrane tensions $\bar{\sigma}$. Compared with the dense data in the energy profiles in our other works on the particle interaction with the cell membrane and vesicle, the data of the energy profiles in the cases of soft elastic particles $\left(\kappa_{1} / \kappa_{2}=1\right.$ and 0.1$)$ in the Letter are relatively sparse in the immediate vicinity of $f=1$. Therefore, the slopes of the energy profiles or the adhesion energy required for the full wrapping states reported in Figs. 3(d) and 3(e) have been underestimated. The corrected version of Figs. 3(d) and 3(e) based on the energy profiles consisting of dense data is shown below (Fig. 1 in this erratum), and for the convenience of the readers, we provide the adopted Figs. 3(b) and 3(c) here as well. The major revision is that the dashed lines representing boundaries between phase II $^{\prime}$ and other phases in the original Figs. 3(d) and 3(e) are shifted rightward.
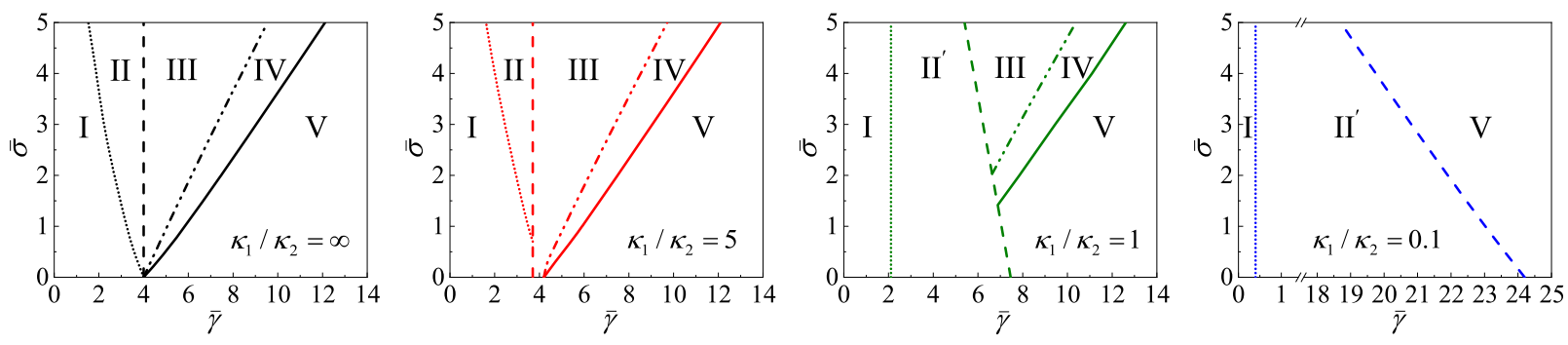

FIG. 1. Wrapping phase diagrams with respect to the normalized adhesion energy $\bar{\gamma}$ and membrane tension $\bar{\sigma}$ at different $\kappa_{1} / \kappa_{2}$ values in three-dimensional cases.

The main conclusions of the Letter remain unchanged. The results including the wrapping phase diagrams in our other work on the particle interaction with the cell membrane and vesicle are based on the energy profiles consisting of dense data and are not affected.

We are grateful to one of the anonymous reviewers of Ref. [1] for bringing this error to our attention.

[1] X. Yi and H. Gao, Incorporation of soft particles into lipid vesicles: Effects of particle size and elasticity, Langmuir 32, 13252 (2016). 